\title{
GROUPS GENERATED BY ELEMENTS WITH RATIONAL FIXED POINTS
}

\author{
by A. W. MASON \\ (Received 31st October 1994)
}

\begin{abstract}
Let $R$ be a commutative integral domain and let $S$ be its quotient field. The group $G L_{2}(R)$ acts on $\hat{S}=S \cup\{\infty\}$ as a group of linear fractional transformations in the usual way. Let $F_{2}(R, z)$ be the stabilizer of $z \in \hat{S}$ in $G L_{2}(R)$ and let $F_{2}(R)$ be the subgroup generated by all $F_{2}(R, z)$. Among the subgroups contained in $F_{2}(R)$ are $U_{2}(R)$, the subgroup generated by all unipotent matrices, and $N E_{2}(R)$, the normal subgroup generated by all elementary matrices.

We prove a structure theorem for $F_{2}(R, z)$, when $R$ is a Krull domain. A more precise version holds when $R$ is a Dedekind domain. For a large class of arithmetic Dedekind domains it is known that the groups $N E_{2}(R), U_{2}(R)$ and $S L_{2}(R)$ coincide. An example is given for which all these subgroups are distinct.
\end{abstract}

1991 Mathematics subject classification: 20H25, 13 F05.

\section{Introduction}

Let $R$ be a commutative integral domain with identity and let $S$ be its quotient field. The group $G L_{2}(S)$ acts on the set $\hat{S}=S \cup\{\infty\}$ as a group of linear fractional transformations in the usual way. Let $g=\left[\begin{array}{ll}a & b \\ c & d\end{array}\right]$ be an element of $G L_{2}(S)$ and let $z \in S$. Then

$$
g(z)=\frac{a z+b}{c z+d}
$$

unless $c \neq 0$ and $z=-d c^{-1}$ in which case $g(z)=\infty$. In addition $g(\infty)=\infty$, when $c=0$. and $g(\infty)=a c^{-1}$, when $c \neq 0$. In this paper we are concerned with those elements of $G L_{2}(R)$ with an $S$-rational fixed point. For each $z \in \hat{S}$ let

$$
F_{2}(R, z)=\left\{g \in G L_{2}(R): g(z)=z\right\}
$$

The subgroup generated by all $F_{2}(R, z)$ is denoted by $F_{2}(R)$. The latter contains a number of important subgroups. Let $X$ be a unipotent matrix in $G L_{2}(R)$. Then by definition $\left(X-I_{2}\right)^{2}=O$ (or, equivalently, $\operatorname{det} X=1$ and $\operatorname{tr} X=2$ ). The simplest unipotent matrices are the elementary matrices $I_{2}+a E_{12}, I_{2}+a E_{21}(a \in R)$. Let $U_{2}(R)$ (resp. $E_{2}(R)$ ) denote the subgroup of $G L_{2}(R)$ generated by all the unipotent (resp. 
elementary) matrices and let $N E_{2}(R)$ be the normal subgroup generated by $E_{2}(R)$. Then we have

$$
E_{2}(R) \leq N E_{2}(R) \leq U_{2}(R) \leq S L_{2}(R)
$$

Another important subgroup of $F_{2}(R)$ is that generated by $F_{2}(R, 0)$ and $F_{2}(R, \infty)$. This is denoted (in Cohn's notation [2, p. 367]) by $G E_{2}(R)$.

To obtain a reasonable structure theorem for $F_{2}(R, z)$ (involving, for example, $R^{*}$, the units of $R$ ) we restrict ourselves to the case where $R$ is a Krull domain. More precise results are established by further restricting to Dedekind domains. Special cases [8], [10] of these results (when $R$ is a particular Dedekind domain contained in a function field) are already known.

For a Dedekind domain $D$ it follows from our results that $U_{2}(D)=F_{2}(D)$ if and only if $D^{*}$ is trivial. How the subgroups in the above chain relate to each other depends very much on $R$. For a large class of arithmetic Dedekind domains they coincide. We provide an example (of an arithmetic Dedekind domain) for which they are all distinct.

\section{General results}

We begin with a number of elementary properties which are true for any integral domain $R$. We recall that $S$ is the quotient field of $R$.

Lemma 1.1. $G E_{2}(R) \cap S L_{2}(R)=E_{2}(R)$.

Proof. This well-known result depends on the fact that $\operatorname{diag}\left(u, u^{-1}\right) \in E_{2}(R)$, for all $u \in R^{*}$. (See, for example, [2, (2.9), p. 370].)

Lemma 1.2. (i) Let $U \in G L_{2}(R)$ be unipotent. Then

$$
U \in F_{2}(R, z)
$$

for some $z \in \hat{S}$.

(ii) $U_{2}(R) \leq F_{2}(R)$.

Proof. By definition

$$
U=\left[\begin{array}{cc}
1+a & b \\
c & 1-a
\end{array}\right]
$$

for some $a, b, c \in R$ with $a^{2}+b c=0$. Then

$$
U \in F_{2}\left(R, z_{0}\right)
$$


where $z_{0}=a c^{-1}$, when $c \neq 0$ and $z_{0}=\infty$, when $c=0$. Part (ii) follows.

Lemma 1.3. (i) Let $g \in G L_{2}(R)$ and $z \in \hat{S}$. Then

$$
g\left(F_{2}(R, z)\right) g^{-1}=F_{2}\left(R, z^{\prime}\right),
$$

where $z^{\prime}=g(z)$.

(ii) $F_{2}(R) \triangleleft G L_{2}(R)$.

Proof. Obvious.

Theorem 1.4. (i) $F_{2}(R, \infty)=\left\{\left[\begin{array}{cc}\alpha & r \\ 0 & \beta\end{array}\right]: \alpha, \beta \in R^{*}, r \in R\right\}$.

(ii) $F_{2}(R, 0)=\left\{\left[\begin{array}{ll}\alpha & 0 \\ r & \beta\end{array}\right]: \alpha, \beta \in R^{*}, r \in R\right\}$.

Proof. Suppose $g=\left(g_{i j}\right) \in G L_{2}(R)$ "fixes" $\infty$ (resp. 0$)$. Then $g_{21}=0\left(\right.$ resp. $\left.g_{12}=0\right)$.

The following consequence is immediate.

Corollary 1.5. Let

$$
N=\left\{\left[\begin{array}{ll}
1 & r \\
0 & 1
\end{array}\right]: r \in R\right\}
$$

and let $M=N^{T}$. Then

(i) $M \cong N \cong R^{+}$

and

(ii) $F_{2}(R, 0) / M \cong F_{2}(R, \infty) / N \cong R^{*} \times R^{*}$.

We extend Corollary 1.5 in the next section.

\section{Krull domains}

To obtain a reasonable version of Theorem 1.4 for any $z \in S$ we assume that our integral domain is integrally closed, which leads us to consider Krull domains.

Let $K$ be a Krull domain with quotient field $L$. By definition [1, Definition 3, Chapter VII, §1.3] there exists a family $\left\{v_{\lambda}: \lambda \in \Omega\right\}$ of discrete valuations on $L$ with the following properties:

(i) $K=\bigcap_{\lambda \in \Omega} V_{\lambda}$,

where $V_{\lambda}$ is the valuation ring of $v_{\lambda}(\lambda \in \Omega)$; 
(ii) for each non-zero $x \in L$,

$$
\left\{\lambda: v_{\lambda}(x) \neq 0\right\}
$$

is a finite subset of $\Omega$.

By an observation made on p. 482 of [1] we may further assume that each $V$, is the localization of $K$ at one of its minimal, non-zero prime ideals. The corresponding $v_{\lambda}$ is called essential. (See [1, Corollary 1, Chapter VII, \$1.4] and [1, Theorem 3, Chapter VII, §1.6].)

Examples of Krull domains include [1, Chapter VII, §1.3] principal ideal domains, unique factorization domains and Dedekind domains.

Theorem 2.1. Let $s \in L^{*}$. An element $g \in F_{2}(K, s)$ if and only if and only if

$$
g=\left[\begin{array}{cc}
\alpha+s c & d \\
c & \beta-s c
\end{array}\right]
$$

where $\alpha, \beta \in K^{*}, c, d \in K, c \in K s^{-1}$ and $d=(\beta-\alpha) s-c s^{2}$.

Proof. Let $g \in F_{2}(K, s)$. We put

$$
g=\left[\begin{array}{ll}
a & b \\
c & d
\end{array}\right], \quad g_{0}=\left[\begin{array}{ll}
0 & -1 \\
1 & -s
\end{array}\right] \quad \text { and } \quad g_{0} g g_{0}^{-1}=\left[\begin{array}{ll}
a^{\prime} & b^{\prime} \\
c^{\prime} & d^{\prime}
\end{array}\right]
$$

Then

$$
a^{\prime}=d+c s, \quad b^{\prime}=-c, c^{\prime}=-b+(d-a) s+c s^{2} \quad \text { and } \quad d^{\prime}=a-c s
$$

Now $g_{0}(s)=\infty$ and so $g_{0} g g_{0}^{-1}(\infty)$. Hence $c^{\prime}=0$. It follows that $a^{\prime}, d^{\prime}$ are the eigenvalues of $g$. Since $K$ is integrally closed [1, Theorem 2(a), Chapter VII, §1.3], we have $a^{\prime}$, $d^{\prime} \in K$. But det $g=a^{\prime} d^{\prime} \in K^{*}$ and so $a^{\prime}, d^{\prime} \in K^{*}$. Put $a^{\prime}=\beta, d^{\prime}=\alpha$.

There remains the question of determining which units of $K$ occur in the entries of a matrix of $F_{2}(K, s)$, as above.

We say that a pair $(\alpha, \beta) \in K^{*} \times K^{*}$ is involved in $F_{2}(K, s)$ if and only if there exist $c, d \in K$, with $c \in K s^{-1}$ such that

$$
d=(\beta-\alpha) s-c s^{2}
$$

Let $\mathfrak{q}$ be any non-zero $K$-ideal and let

$$
\mathfrak{q}^{-1}=(K: \mathfrak{q})=\{x \in L: x \mathfrak{q} \leq K\}
$$

By definition $\mathfrak{q q}^{-1} \leq K$. The ideal $\mathfrak{q}$ is called invertible if and only if $\mathfrak{q q} \mathfrak{q}^{-1}=K$. (See [1, Proposition 10, Chapter II, §5.6].) 
Corollary 2.2. Let $s=u / v$, where $u, v \in K$ and $u, v \neq 0$, and let $s=u K+v K$. The pair $(\alpha, \beta) \in K^{*} \times K^{*}$ is involved in $F_{2}(K, s)$ if and only if

$$
\alpha \equiv \beta\left(\bmod s^{-1}\right) .
$$

Proof. Suppose that $(\alpha, \beta)$ is involved in $F_{2}(K, s)$. Let $\gamma=\beta-\alpha$. Then there exist $c, d, e \in K$ such that

$$
c u=e v \text { and } c u^{2}=\gamma u v-d v^{2}
$$

Hence $\gamma=x u+y v$, where $x=c v^{-1}$ and $y=d u^{-1}$. Clearly $x v, y u \in K$. In addition $x u=e \in K$ and $y v=\gamma-e \in K$. It follows that $\gamma \in \mathbf{s s}^{-1}$.

Conversely suppose that $\gamma=\beta-\alpha \in \mathfrak{s s}^{-1}$. Then there exist $a, b \in \mathfrak{s}^{-1}$ such that

$$
\gamma=a u-b v
$$

By Theorem 2.1

$$
\left[\begin{array}{cc}
\alpha+a u & -b u \\
a v & \beta-a u
\end{array}\right] \in F_{2}(K, s)
$$

Among the normal subgroups of $F_{2}(K, s)$ the most important are

(i) The centre of $G L_{2}(K)$,

$$
Z=\left\{\alpha I_{2}: \alpha \in K^{*}\right\}
$$

and

(ii) $U_{2}(K, s)=\left\{\left[\begin{array}{cc}1+s c & -s^{2} c \\ c & 1-s c\end{array}\right]: c \in K \cap K s^{-1} \cap K s^{-2}\right\}$.

$U_{2}(K, s)$ consists of all the unipotent matrices in $F_{2}(K, s)$. It is clear that

$$
U_{2}(K, s) \cong H^{+},
$$

where $H=K \cap K s^{-1} \cap K s^{-2}$.

The following is an immediate consequence of Theorem 2.1.

Corollary 2.3. With the above notation, let

$$
C(\mathfrak{s})=\left\{\alpha \in K^{*}: \alpha \equiv 1 \quad\left(\bmod s^{-1}\right)\right\}
$$

and let

$$
M=Z \cdot U_{2}(K, s)
$$


Then

$$
F_{2}(K, s) / M \cong C(\mathfrak{s})
$$

For many Krull domains of course (e.g. Dedekind domains) we always have $\mathfrak{s s}^{-1}=K$. Examples for which $\mathfrak{s s}^{-1} \neq K$ are however easy to find. Let $K=F(x, y)$, the polynomial ring in $x, y$ over any field $F$. Then, when $u=x$ and $v=y$,

$$
K / \mathfrak{s s}^{-1} \cong F \text {. }
$$

\section{Dedekind domains}

It is clear that a more precise version of Theorem 2.1 holds for those Krull domains in which every 2-generated (and hence every finitely generated) non-zero ideal is invertible. (An integral domain with the latter property is called a Prüfer domain [3, Chapter IV].) By [3, (43.16) Theorem] such a Krull domain is Dedekind. (Gilmer's proof of this result makes use of the fact that the valuations in the defining family for the Krull domain can be assumed to be essential.)

Throughout this section $D$ denotes a Dedekind domain and $E$ is its quotient field. Theorem 2.1 and Corollary 2.2 extend to the following.

Theorem 3.1. For each $s \in E^{*}$,

$F_{2}(D, s)=\left\{\left[\begin{array}{cc}\alpha+s c & d \\ c & \beta-s c\end{array}\right]: \alpha, \beta \in D^{*}, \quad c \in D \cap D s^{-1}, \quad d \in D, \quad d=(\beta-\alpha) s-c s^{2}\right\}$.

\section{Corollary 3.2.}

$$
F_{2}(D, s) / U_{2}(D, s) \cong D^{*} \times D^{*}
$$

Corollary 3.3.

$$
F_{2}(D)=U_{2}(D) \text { if and only if } D^{*}=\{1\} .
$$

Let $C$ be a (suitable) projective curve over a field $k$ and let $P$ be one of its closed points. Let $\mathcal{C}=\mathcal{C}(C, P, k)$ be the coordinate ring of the affine curve obtained by removing $P$ from $C$. Then $\mathcal{C}$ is a Dedekind domain [12, p. 96] contained in the function field, $F$, of $C$. As a Krull domain $\mathcal{C}$ is defined by the family of all discrete valuations on $F$, trivial on $k$, other than that corresponding to $P$. The simplest case is the polynomial ring $\mathcal{C}=k[t]$.

Theorem 3.1 is known [8, Lemma 1.8] and [10, Lemma 2(ii)] for the case $D=\mathcal{C}$.

The orbits of $\hat{E}$ modulo $G L_{2}(D)$ (or $S L_{2}(D)$ ) can be identified with the elements of the ideal class group of $D$. As a consequence any pair of elements of $\hat{E}$ in the same $G L_{2}(D)$-orbit are also in the same $S L_{2}(D)$-orbit. This appears to be well-known. Serre, for example, [11, p. 507] states this result (without proof) in a rather different form. We provide such a proof. 
By [1, Proposition 12, Chapter II, $\$ 5.7]$ the ideal class group of $D$ can be identified with its Picard group, $\operatorname{Pic}(D)$. We recall the following definition of $\operatorname{Pic}(D)$.

Non-zero $D$-ideals $\mathfrak{q}_{1}, \mathfrak{q}_{2}$ are equivalent, if and only if

$$
x \mathfrak{q}_{1}=y \mathfrak{q}_{2},
$$

for some non-zero $x, y \in D$. This is an equivalence relation and the equivalence classes form an abelian group under multiplication in the usual way, with the identity as the class consisting of the principal ideals. This group is isomorphic to $\operatorname{Pic}(D)$.

Let $t \in E$. Then $t=u v^{-1}$, for some $u, v \in D$, with $v \neq 0$. We denote by $I(t)$ the image in $\operatorname{Pic}(D)$ of the $D$-ideal

$$
u D+v D
$$

We define $I(\infty)$ to be the identity of $\operatorname{Pic}(D)$. It is clear that we have a well-defined map

$$
\phi: \hat{E} \rightarrow \operatorname{Pic}(D)
$$

given by

$$
\phi(t)=I(t)
$$

Theorem 3.3. (i) $\phi$ is surjective.

(ii) For all $t_{1}, t_{2} \in \hat{E}$,

$$
\phi\left(t_{1}\right)=\phi\left(t_{2}\right) \Leftrightarrow t_{2}=g\left(t_{1}\right), \quad \text { for some } g \in S L_{2}(D)
$$

Proof. Part (i) follows from the classical result that every $D$-ideal has at most two generators.

For part (ii) it is obvious that, if $t_{2}=g\left(t_{1}\right)$ where $g \in G L_{2}(D)$, then $\phi\left(t_{1}\right)=\phi\left(t_{2}\right)$. Suppose then that

$$
\phi\left(t_{1}\right)=\phi\left(t_{2}\right)
$$

We consider first the care where $t_{1}$ or $t_{2}$ is 0 or $\infty$. Since $\phi(0)=\phi(\infty)$ we may assume that $t_{1}=\infty$ and that $t_{2}=u v^{-1}$, where $u, v \in D$ and $u, v \neq 0$.

Since $u D+v D$ is a principal ideal, $\exists x, y \in D$ such that

(i) $x D+y D=D$,

(ii) $x u+y v=0$.

Let $g_{0}$ be any element of $S L_{2}(D)$ with second row $(x, y)$. Then

$$
g_{0}\left(t_{2}\right)=\infty
$$

We assume from now on that $t_{1}=u_{1} / v_{1}$ and $t_{2}=u_{2} / v_{2}$, where $u_{1}, v_{1}, u_{2}, v_{2} \in D$ are all non-zero. Now by the definition of $\operatorname{Pic}(D)$

$$
a\left(u_{1} D+v_{1} D\right)=b\left(u_{2} D+v_{2} D\right)
$$


for some non-zero $a, b \in D$. We may assume therefore that $a=b=1$.

Let

$$
I=u_{1} D+v_{1} D=u_{2} D+v_{2} D .
$$

Since $I I^{-1}=D$, there exist $u_{i}^{\prime}, v_{i}^{\prime} \in I^{-1}$ such that

$$
u_{i} u_{i}^{\prime}+v_{i} v_{i}^{\prime}=1 \quad(i=1,2) .
$$

Let

$$
g_{i}=\left[\begin{array}{cc}
-u_{i}^{\prime} & -v_{i}^{\prime} \\
v_{i} & -u_{i}
\end{array}\right] \quad(i=1,2)
$$

Then $g_{i}\left(t_{i}\right)=\infty(i=1,2)$ and so

$$
g_{2}^{-1} g_{1}\left(t_{1}\right)=t_{2}
$$

But $g_{2}^{-1} g_{1} \in S L_{2}(D)$

Corollary 3.4. There exist one-one correspondences

$$
\hat{E} / S L_{2}(D) \Leftrightarrow \hat{E} / G L_{2}(D) \Leftrightarrow \operatorname{Pic}(D)
$$

Proof. Immediate from Theorem 3.3.

We represent the elements of $\operatorname{Pic}(D)$ by representatives of the orbits of $\hat{E}$, modulo $S L_{2}(D)$,

$$
\infty \text { and } s_{\lambda} \quad(\lambda \in \Lambda)
$$

where $s_{i} \in E^{*}$.

Corollary 3.5. For all $z \in \hat{E}, \exists g \in S L_{2}(D)$ such that

$$
g F_{2}(D, z) g^{-1}=F_{2}\left(D, z^{*}\right)
$$

for some unique $z^{*} \in\{\infty\} \cup\left\{s_{\lambda}: \lambda \in \Lambda\right\}$

Proof. The existence of $z^{*}$ follows from Theorem 3.3 and Lemma 1.3(i). For uniqueness we have to show that, for all $z_{1}, z_{2} \in \hat{E}$,

$$
F_{2}\left(D, z_{1}\right)=F_{2}\left(D, z_{2}\right) \Rightarrow z_{1}=z_{2}
$$

Choose $g \in S L_{2}(D)$ such that $g\left(z_{1}\right), g\left(z_{2}\right) \neq \infty$. By Lemma 1.3(i) we may assume that $z_{1}, z_{2} \neq \infty$. Then $z_{1}=u / v$, for some $u, v \in D, v \neq 0$. 
It is easily verified that

$$
g_{0}=\left[\begin{array}{cc}
1+u v & -u^{2} \\
v^{2} & 1-u v
\end{array}\right] \in F_{2}\left(D, z_{1}\right)
$$

Then $g_{0}\left(z_{2}\right)=z_{2}$ which implies that $z_{1}=z_{2}$.

A special case of Corollary 3.5 is the following.

Corollary 3.6. Let $D$ be a principal ideal domain.

(i) For all $z \in \hat{E}$, there exists $g \in S L_{2}(D)$ such that

$$
g F_{2}(D, z) g^{-1}=F_{2}(D, \infty)
$$

(ii) $N E_{2}(D)=U_{2}(D)$

Proof. Part (i) follows from Corollary 3.5 since $\operatorname{Pic}(D)=\{\infty\}$.

Part (ii) follows from part (i) and Lemma 1.2(i).

\section{Examples}

\section{1. $G E_{2}$-rings}

Let $R$ be a $G E_{2}$-ring. Then by definition

$$
G L_{2}(R)=G E_{2}(R)\left(=F_{2}(R)\right) .
$$

By Lemma 1.1 it follows that

$$
S L_{2}(R)=E_{2}(R)
$$

and hence that

$$
E_{2}(R)=N E_{2}(R)=U_{2}(R)=S L_{2}(R) \cap F_{2}(R) .
$$

Examples of $G E_{2}$-rings include all Euclidean domains, e.g. $\mathbb{Z}$, the ring of rational integers, $k[t]$, where $k$ is a field, and $\mathbb{Z}[i]$, where $i^{2}=-1$. Euclidean domains are, of course, principal ideal domains. There are however many $G E_{2}$-rings which are not principal ideal domains.

Let $K_{0}$ be a global field (i.e. a finite, separable extension of $\mathbb{Q}$, the set of rational numbers, or $k(t)$, where $k$ is finite field) and let $S$ be a finite set (of equivalence classes) of valuations of $K_{0}$, which includes all the archimedean valuations. Let

$$
A_{S}=\left\{x \in K_{0}: v(x) \geq 0, \quad \text { for all } v \notin S\right\}
$$


Then $A_{S}$ is a Dedekind domain [11, p. 189], called the ring of $S$-integers of $K_{0}$. (The valuations of $K_{0}$ not in $S$ are a defining family for $A_{S}$.) From a theorem of Dirichlet it is known that $A_{S}^{*}$ is finite if and only if card $(S)=1$. The latter condition is only satisfied in three cases (two of which are $A_{s}=\mathbb{Z}$ and $A_{s}=\mathcal{C}(C, P, k)$, where $k$ is finite). Liehl [5], using previous results of Vaserstein [15], has proved that when $A_{S}^{*}$ is infinite, $A_{s}$ is a $G E_{2}$-ring. Examples of $A_{s}$ with infinite $A_{s}^{*}$, which are not principal ideal domains, can be found, for example, among the rings of integers of real quadratic extensions of $\mathbb{Q}$.

\subsection{The Bianchi groups}

The purpose of this example is to record the existence of a class of Dedekind domains $D$, for which $U_{2}(D) \neq N E_{2}(D)$ precisely when $D$ is not a principal ideal domain. (See Corollary 3.6.)

The Bianchi groups are the groups $S L_{2}\left(\mathcal{O}_{d}\right)$ (or $P S L_{2}\left(\mathcal{O}_{d}\right)$ ), where $\mathcal{O}_{d}$ is the ring of integers of the imaginary quadratic number fielded $\mathbb{Q}(\sqrt{ }-d)$, with $d$ a positive, squarefree integer.

It follows from a result of Serre [11, Corollaire 3, p. 516], as shown by Grunewald, Mennicke and Vaserstein [4, p. 189], that

$$
U_{2}\left(\mathcal{O}_{d}\right) \neq N E_{2}\left(\mathcal{O}_{d}\right)
$$

when $\mathcal{O}_{d}$ is not a principal ideal domain. It is well-known [13] that $\mathcal{O}_{d}$ is a principal ideal domain if and only if $d=1,2,3,7,11,19,43,67$ and 163 .

It is also known [7] that

$$
S L_{2}\left(\mathcal{O}_{d}\right) \neq U_{2}\left(\mathcal{O}_{d}\right)
$$

for all but finitely many $d$.

\subsection{An example of Serre}

We conclude with an example of a Dedekind domain $D$ for which the groups $E_{2}(D), N E_{2}(D), U_{2}(D)$ and $S L_{2}(D)$ are all distinct.

A structure theorem of Serre [12, Theorem 10, p. 119] for the group $G L_{2}(\mathcal{C})$ provides a source of many examples of this type. We describe a particular case.

Let $k_{0}=G F(2)$ and let

$$
f(t)=t^{4}+t+1 \quad\left(\in k_{0}[t]\right)
$$

We put

$$
\mathcal{A}=\left\{\frac{g}{f^{s}}: g \in k_{0}[t], s \in \mathbb{Z}, s \geq 0, \operatorname{deg} g \leq 4 s\right\}
$$


In the above notation we can write

$$
\mathcal{A}=\mathcal{C}\left(C_{0}, P_{0}, k_{0}\right)
$$

where $C_{0}$ is the projective line over $k_{0}$ and $P_{0}$ is its point corresponding to the (irreducible) polynomial $f$. It follows that $\mathcal{A}$ is an arithmetic Dedekind domain. It is clear that

$$
\mathcal{A}^{*}=k_{0}^{*}=\{1\},
$$

and hence that

$$
G L_{2}(\mathcal{A})=S L_{2}(\mathcal{A}), \quad U_{2}(\mathcal{A})=F_{2}(\mathcal{A})
$$

It is known (see, for example, [8, Theorem 3.4(ii)]) that

$$
\operatorname{Pic}(\mathcal{A}) \cong \mathbb{Z} / 4 \mathbb{Z}
$$

(i) $E_{2}(\mathcal{A}) \neq N E_{2}(\mathcal{A})$. Since $\mathcal{A}$ is not a principal ideal domain it follows from a result of Suslin [14] that

$$
E_{2}(\mathcal{A}) \propto S L_{2}(\mathcal{A})
$$

(ii) $N E_{2}(\mathcal{A}) \neq U_{2}(\mathcal{A})$. The author $[9$, Theorem 3.3(ii)] has proved the existence of an epimorphism

$$
r: U_{2}(\mathcal{A}) / N E_{2}(\mathcal{A}) \rightarrow V^{+} * V^{+} * V^{+}
$$

where $V^{+}$is the additive group of a (countably) infinite dimensional vector space $V$ over $k_{0}$.

(iii) $U_{2}(\mathcal{A}) \neq S L_{2}(\mathcal{A})$. The group $S L_{2}(\mathcal{A})$ acts on a tree $X$ (whose vertices are the equivalence classes of lattices of the vector space $\left(k_{0}\right)^{2}$.) (See [12, Chapter II].) Serre $\left[12,2.4 .2(\mathrm{c})\right.$, p. 113] has determined the structure of the quotient graph $S L_{2}(\mathcal{A}) \backslash X$. It is known (see, for example, [8, Theorems 1.11, 1.12]) that every unipotent matrix in $S L_{2}(\mathcal{A})$ is conjugate to an element which fixes one of the vertices of $X$. From the fundamental theorem of the theory of groups acting on trees [12, Theorem 13, p. 55] it follows that there exists on epimorphism from $S L_{2}(\mathcal{A})$ to the fundamental group of the graph $S L_{2}(\mathcal{A}) \backslash X$, whose kernel contains $U_{2}(\mathcal{A})$. Hence there exists an epimorphism

$$
\sigma: S L_{2}(\mathcal{A}) / U_{2}(\mathcal{A}) \rightarrow F_{2}
$$

where $F_{2}$ is the free group of rank 2 . 


\section{REFERENCES}

1. N. Bourbaki, Commutative algebra (Hermann, Paris, 1972).

2. P. M. CoHN, On the structure of the $G L_{2}$ of a ring, Publ. Math. I.H.E.S. 30 (1966), 365-413.

3. R. Gilmer, Multiplicative Ideal Theory in Queen's Papers in Pure and Applied Mathematics (volume 90, Kingston, Ontario, Canada, 1992).

4. F. Grunewald, J. Mennicke and L. Vaserstein, On the groups $S L_{2}(\mathbb{Z}[x])$ and $S L_{2}(k[x, y])$, Israel J. Math. 86 (1994), 157-193.

5. B. LIEHL, On the group $S L_{2}$ over orders of arithmetic type, J. Reine Angew. Math. 323 (1981), 345-358.

6. A. W. Mason, Free quotients of congruence subgroups of $S L_{2}$ over a Dedekind ring of arithmetic type contained in a function field, Math. Proc. Cambridge Philos. Soc. 101 (1987), 421-429.

7. A. W. MASON, Free quotients of the Bianchi groups and unipotent matrices, submitted for publication.

8. A. W. MASON, Free quotients of congruence subgroups of $S L_{2}$ over a coordinate ring and unipotent matrices, submitted for publication.

9. A. W. MaSon, Unipotent matrices, modulo elementary matrices, in $S L_{2}$ over a coordinate ring, submitted for publication.

10. W. RadtKe, Diskontinuierliche arithmetische Gruppen im Funktionenkörperfall, J. Reine Angew. Math. 363 (1985), 191-200.

11. J.-P. SerRe, Le problème des groupes de congruence pour $S L_{2}$, Ann. of Math. 92 (1970), 489-527.

12. J.-P. SERRE, Trees (Springer-Verlag, Berlin, 1980).

13. H. M. STARK, A complete determination of the complex quadratic fields of class-number one, Michigan Math. J. 14 (1967), 1-27.

14. A. A. Suslin, One theorem of Cohn, J. Soviet Math. 17 (1981), 1801-1803.

15. L. N. VASERSTEIN, On the group $S L_{2}$ over Dedekind rings of arithmetic type, Math. USSR - Sb. 18 (1972), 321-332.

Department OF Mathematics

UNIVERSITY OF GLASGOW

GLASGOW

G12 8QW

E-mail address: awm@MATHS.GLA.AC.UK 